\title{
SELECTIVE WITHDRAWAL FROM STRATIFIED STREAMS
}

\author{
CHIA-SHUN YIH ${ }^{1}$ and SONGPING ZHU ${ }^{2}$
}

(Received 19 May 1994; revised 23 November 1994)

\begin{abstract}
Selective withdrawal from a stratified stream is considered. The average density of the withdrawn fluid and the flow pattern are found, within the limitations on the densimetric Froude number and the withdrawal rate specified in this paper, to depend on the strength and location of the sink, and very little on any slight variation in the velocity distribution far upstream and the densimetric Froude number. The upstream density distribution is assumed linear, but many other density distributions can be similarly treated.
\end{abstract}

\section{Introduction}

Power plants often draw their cooling water from a stream stratified in temperature and therefore in density. Thus arises the interest in the problem of selective withdrawal. The same problem is encountered when water is drawn from an estuary stratified in salinity, and the smallest average salinity of the withdrawn water is desired, or else when pollutants form a layer in a stream and are to be removed.

The problem of selective withdrawal was initiated by the theoretical work of Craya [1] and the experimental work of Gariél [3]. Craya used Richardson's formula [6] to obtain an exact solution of the problem of two-dimensional flow into a sink. The flowing fluid is bounded below by an interface with a stagnant heavier fluid and above by two planes symmetrically inclined at an angle $\theta=\pi / 3$ with the vertically upward direction and intersecting at the sink. This solution is exact and elegant, and has the advantage that the fluids is at rest at infinity, so that the velocity discontinuity along the interface has been developed spatially from rest if not temporally from a state of rest everywhere. Craya postulated that the densimetric Froude number $F$ (based on an appropriately reduced gravitational acceleration) obtained in his solution is critical, in the sense that if the number is exceeded the lower fluid will flow into the sink. This

\footnotetext{
${ }^{1}$ Dept of Mech. Eng. and Appl. Mech., The University of Michigan, Ann Arbor, Michigan 48109, USA.

${ }^{2}$ Dept of Mathematics, The University of Wollongong, Wollongong, NSW 2522, Australia.

(C) Australian Mathematical Society, 1996, Serial-fee code 0334-2700/96
} 
postulate has never been proved but is plausible. To apply his solution to Gariél's experiment made in a channel with a horizontal bottom, Craya made a rough estimate of the critical $F$ for Gariél's flow from the result of his solution and obtained the value 0.75 for it. Gariél's experimental result agrees with this estimate fairly well.

Craya's work has been extended in recent years to include other values of $\theta$, which is $\pi / 3$ in Craya's case. But the later workers have preferred to invert the geometry and eliminate the stagnant fluid above the interface. Then the interface becomes a free surface and the densimetric Froude number becomes the ordinary Froude number. Tuck and Vanden-Broeck [7] studied the case of $\theta=0$ (corresponding to a semiinfinite fluid). Hocking [4] studied the cases $0 \leq \theta \leq \pi / 2$. He found only one value for $F$ for each $\theta$, including $\theta=\pi / 2$. Then Vanden-Broeck and Keller [8] studied the cases for $0 \leq \theta \leq \pi / 2$. Unique values of $F$ (in agreement with Hocking's results if attention is given to the difference between their definitions of the Froude number) were found for all these values of $\theta$. All these solutions, like Craya's, are physically significant because the flow originates from infinity, where the fluid is at rest, provided $\theta<\pi / 2$.

For the sink located at the bottom of the channel and $\theta=\pi / 2$, Vanden-Broeck and Keller [8] obtained the Froude number 1 by making $\theta$ approach $\pi / 2$ from below. This is the critical Froude number for the case of channel flow. For $\theta=\pi / 2$, however, they found a range of possible values for $F$ from some minimum value to infinity. Since the VBK solutions apply to flows under a stagnant layer, for a discussion of the significance of these supercritical solutions (for which $F>1$ ) let us revert to the existence of an upper stagnant layer. For $F>1$ the upper fluid will probably enter the sink in spite of the existence of the supercritical solutions, much as a fluid of uniform density will not separate to give a stagnant layer (at an infinite $F$ in that case), in spite of the existence of the classical free-streamline solution. (In the latter case separation would violate Kelvin's theorem on the persistence of irrotationality if the flow is established from rest, and all the fluid will flow into the sink, allowing no separation.) The physical significance of the supercritical solutions has to be decided by an examination of their stability. Instability of such flows is implicit in Craya's postulate. The VBK supercritical solutions do not contradict Craya's postulate. Indeed, the semi-infinite range of possible supercritical Froude numbers surely suggests their lack of physical significance. Thus the VBK supercritical solutions have gone a long way to support, if not quite to prove, Craya's postulate. Furthermore, the Froude number 1 obtained by making $\theta$ approach $\pi / 2$ from below, in agreement with Hocking's result, is exactly what Craya sought to obtain for Gariél's experiment.

The problem of flows of a continuously stratified fluid in a channel into a sink was treated by Yih [9], who found solutions without separation for $F$, the densimetric Froude number, above $1 / \pi$. As $F$ approaches $1 / \pi$ from above, however, a more and more elongated eddy occurs, in which there is inevitably density inversion, suggesting 
that even before $F$ reaches $1 / \pi$ fluid separation will take place. This led Debler [2] to do his pioneering work. He found that when $F$ is low the flow separates into a stagnant and a flowing region. As $F$ decreases the depth of the flowing region also decreases, in such a way that $F^{\prime}$, the densimetric Froude number based on the depth of the flowing region, remains constant. Debler's experiment showed that constant to be 0.24 . The constancy of $F^{\prime}$ can be expected on theoretical grounds. Kao [5], by numerical computation, found that

$$
0.33 \leq F^{\prime} \leq \infty .
$$

Only the number 0.33 is significant, much as in the problem of two fluid layers. The figure 0.33 is consistent with Yih's results [9], and is of the same order of magnitude as Debler's experimental value 0.24. The difference between the two figures is understandable, since a velocity discontinuity cannot be achieved with a viscous fluid, and the viscosity will always thicken a flowing layer and bring down the value of $F^{\prime}$.

The foregoing account of the history of the problem of two-dimensional selective withdrawal points to the lack of attention to the effect of a parallel flow (in addition to the flow caused by the sink) on selective withdrawal. This paper addresses this lack, and gives solutions for selective withdrawal from a stratified stream, which are applicable to a wide range of problems of practical interest.

\section{Analysis}

We shall consider first the case of a uniform velocity $U$ of the stream, of depth $d$, before the sink, of strength (volume flux) $\hat{q}$, is placed in the fluid. This case corresponds to that of a sink moving in an otherwise quiescent fluid. The upper surface is free, but is practically flat for the problem considered here, so that we can assume the flow to be confined between two parallel rigid boundaries at distance $d$ apart. Such an assumption is consistent with the assumption of small $\beta$ made later. The upstream velocity after the sink is introduced will be derived. Assuming that the sink strength is built from zero to $\hat{q}$ in a large (compared with $d^{2} / \hat{q}$ ) period $T$, and that during that period the flow far upstream is horizontal, we have, for the region far upstream and with subscripts indicating partial differentiation,

$$
\rho u_{t}=-p_{x}, \quad 0=-p_{y}-g \rho,
$$

where $\rho$ is the density, $u$ is the horizontal component of the velocity, $p$ is the pressure, $g$ is the gravitational acceleration, $t$ is the time, and $x$ and $y$ are Cartesian coordinates. We measure $x$ from the position of the sink, and $y$ from the horizontal bottom of the channel. The density far upstream is assumed to be

$$
\rho=\rho_{0}\left(1-\beta d^{-1} y\right)
$$


where $\beta \ll 1$ for all cases of practical interest. Yih [10] gave infinitely many upstream density distributions which, with corresponding upstream velocity distributions, allow the partial differential equation governing stratified flows to become linear. These pairs of upstream distributions, and others differing little therefrom, can be treated in a way similar to that given here.

Eliminating $p$ from (1), we have, since $\rho$ is a function of $y$ only,

$$
(\rho u)_{t y}=0 \text {, }
$$

the solution of which is

$$
\rho u=f(y)+k(t)
$$

where

$$
k(0)=0, \quad k^{\prime}(T)=0, \quad k(T)=\alpha \rho_{0} .
$$

Initially the velocity is $U$, so that (3) can be written as

$$
u=U+\alpha \rho_{0} / \rho
$$

and this, with $\rho$ given by (2), gives

$$
u=U+\alpha\left(1+\beta d^{-1} y\right)+\mathrm{O}\left(\beta^{2}\right) .
$$

The constant $\alpha$ is related to $\hat{q}$. Integrating (5), we have

$$
U d+\hat{q}=U d+\alpha d(1+\beta / 2)+\mathrm{O}\left(\beta^{2}\right),
$$

from which we obtain, upon neglecting terms of $O\left(\beta^{2}\right)$,

$$
\alpha=(\hat{q} / d)(1-\beta / 2) .
$$

Then

$$
u=U+\hat{q} / d-(\beta \hat{q} / d)(1 / 2-y / d) .
$$

We shall make things dimensionless by using $U+\hat{q} d^{-1}$ and $d$ as the scales of velocity and length, respectively. Then (7) becomes

$$
u=1-\beta q(1 / 2-y),
$$

and the stream function far upstream is obtained from this by integration to be

$$
\psi=y-(\beta q / 2)\left(y-y^{2}\right) .
$$


In (8) and (9), $q$ is defined by

$$
q=\hat{q} /(U d+\hat{q}) .
$$

From (9), upon neglecting terms of $O\left(\beta^{2}\right)$ we have

$$
y=\psi+(\beta q / 2)\left(\psi-\psi^{2}\right) .
$$

To obtain the associated stream function $\psi^{\prime}$ used by Yih [9], we note that far upstream

$$
\rho=\rho_{0}(1-\beta y)
$$

and

$$
\psi^{\prime}=\int_{0}^{\psi}\left(\rho / \rho_{0}\right)^{1 / 2} d \psi=\psi-\beta \psi^{2} / 4
$$

upon neglecting $\mathrm{O}\left(\beta^{2}\right)$. From (13) and (9) one obtains, for $x=-\infty$,

$$
\psi^{\prime}=y-(\beta / 2)\left[q y+(1 / 2-q) y^{2}\right] .
$$

This will later be denoted by $\psi_{0}^{\prime}$. From (14) we have

$$
y=\psi^{\prime}+(\beta / 2)\left[q \psi^{\prime}+(1 / 2-q) \psi^{\prime 2}\right] .
$$

Then at $x=-\infty$,

$$
\frac{1}{\rho_{0}} \frac{d \rho}{d \psi^{\prime}}=-\beta \frac{d y}{d \psi^{\prime}}=-\beta-\left(\beta^{2} / 2\right)\left[q+(1-2 q) \psi^{\prime}\right],
$$

with terms of $\mathrm{O}\left(\beta^{3}\right)$ neglected.

The equation governing $\psi^{\prime}$ has been given by Yih [9]. In dimensionless terms, it is

$$
\nabla^{2} \psi^{\prime}+F_{0}^{-2} \frac{d\left(\rho / \rho_{0}\right)}{d \psi^{\prime}} y=h\left(\psi^{\prime}\right)
$$

in which

$$
\nabla^{2}=\partial^{2} / \partial x^{2}+\partial^{2} / \partial y^{2}, \quad F_{0}^{2}=\left(U+\hat{q} d^{-1}\right)^{2} /(g d),
$$

and $d \rho / d \psi^{\prime}$ is a function of $\psi^{\prime}$ only, given by (16). The function $h\left(\psi^{\prime}\right)$ is to be determined from the upstream conditions. We define a densimetric Froude number $F$ by

$$
F^{-2}=\beta F_{0}^{-2}
$$


and shall regard $F^{-2}$ as of $O(1)$. Substituting (14), (15), and (16) into (17), we have

$$
h\left(\psi^{\prime}\right)=-(\beta / 2)(1-2 q)-F^{-2}\left[(1+\beta q) \psi^{\prime}+(3 \beta / 4)(1-2 q) \psi^{2}\right] .
$$

Then (17) becomes

$$
\begin{aligned}
\nabla^{2} \psi^{\prime} & -F^{-2}\left\{1+(\beta / 2)\left[q+(1-2 q) \psi^{\prime}\right]\right\} y \\
& =-(\beta / 2)(1-2 q)-F^{-2}\left[(1+\beta q) \psi^{\prime}+(3 \beta / 4)(1-2 q) \psi^{\prime 2}\right] .
\end{aligned}
$$

Let the $\psi^{\prime}$ far upstream, given by (14), be denoted by $\psi_{0}^{\prime}$. Since (20) has been obtained from the upstream conditions, it is obvious that $\psi_{0}^{\prime}$ should satisfy (21), as can be verified. We shall then write

$$
\psi^{\prime}=\psi_{0}^{\prime}+\phi
$$

and substitute it into (21) to obtain, after some cancellations,

$$
\begin{aligned}
\nabla^{2} \phi & -(1 / 2) \beta F^{-2}(1-2 q) \phi y \\
& =-F^{-2}\left\{\phi+\beta\left[q \phi+(3 / 4)(1-2 q) \phi\left(\phi+2 \psi_{0}^{\prime}\right)\right]\right\} .
\end{aligned}
$$

The $\phi$ corresponds to the flow induced by the sink. It is therefore proportional to $\hat{q}$ and therefore of the order of $q$, which, as will be seen later, will be restricted to $1 / 7$. Ignoring terms of $\mathrm{O}(\beta q)$ in (23) in comparison with those of $\mathrm{O}(q)$, we have

$$
\nabla^{2} \phi+F^{-2} \phi=0
$$

For the reasons that will be explained later we shall assume

$$
F>1 / \pi
$$

The solution of (24) is then

$$
\begin{array}{ll}
\phi=\sum_{n=1}^{\infty} A_{n} \sin n \pi y \exp \left(n^{2} \pi^{2}-F^{-2}\right)^{1 / 2} x & \text { for } x<0, \\
\phi=\phi_{0}+\sum_{n=1}^{\infty} B_{n} \sin n \pi y \exp \left[-\left(n^{2} \pi^{2}-F^{-2}\right)^{1 / 2} x\right] & \text { for } x \geq 0,
\end{array}
$$

where $\phi_{0}$ will be specified. Matching $\phi_{x}$ and $\phi$ at $x=0$ we have

$$
\begin{aligned}
& A_{n}=-B_{n}, \\
& A_{n}=\int_{0}^{1} \phi_{0}(y) \sin n \pi y d y .
\end{aligned}
$$


If the sink is located at $(0,0)$,

$$
\phi_{0}(y)=\frac{q \sin \left[F^{-1}(1-y)\right]}{\sin F^{-1}} .
$$

If it is located at $\left(0, y_{s}\right)$,

$$
\begin{array}{ll}
\phi_{0}(y)=\phi_{+}=A q \sin \left[F^{-1}(1-y)\right] & \text { for } y_{s}<y \leq 1, \\
\phi_{0}(y)=\phi_{-}=B q \sin F^{-1} y & \text { for } 0 \leq y<y_{s},
\end{array}
$$

where $A$ and $B$ in these equations are determined by the two conditions

(i) $d \phi_{0} / d y$ must be continuous at $y=y_{s}$,

(ii) $\phi_{+}\left(y_{s}\right)-\phi_{-}\left(y_{s}\right)=q$.

Thus

$$
\begin{aligned}
& -A \cos \left[F^{-1}\left(1-y_{s}\right)\right]=B \cos F^{-1} y_{s}, \\
& A \sin \left[F^{-1}\left(1-y_{s}\right)\right]-B \sin F^{-1} y_{s}=1 .
\end{aligned}
$$

Once $A$ and $B$ are determined, we can go far upstream to find $y_{2}$ and $y_{1}$ where

$$
\psi^{\prime}\left(y_{2}\right)=\psi_{0}^{\prime}\left(y_{s}\right)+\phi_{+}\left(y_{s}\right), \quad \psi^{\prime}\left(y_{1}\right)=\psi_{0}^{\prime}\left(y_{s}\right)+\phi_{-}\left(y_{s}\right) .
$$

These formulae bear directly on the problem of pollutant removal. For a given $F$, we determine $q$ and $y_{s}$ to obtain the $y_{2}$ and $y_{1}$ specified (some iteration may be necessary). When $y_{1}=0$, we have $y_{s}=0$, and the determination of $q$ to obtain the specified $y_{2}$ is simple.

For a sink located at the bottom, the average density $\bar{\rho}$ of the fluid withdrawn is

$$
\bar{\rho}=q^{-1} \int_{0}^{\psi_{2}} \rho d \psi
$$

the integration being done at a section far upstream. For a linearly stratified fluid, the use of (12) gives

$$
\bar{\rho}=\rho_{0}(1-\beta q / 2),
$$

if terms of $\mathrm{O}\left(\beta^{2}\right)$ are neglected. For a sink located at $\left(0, y_{s}\right)$, we note first that $\psi_{2}-\psi_{1}=q$. Then, using (11), we have

$$
\begin{aligned}
\bar{\rho} & =q^{-1} \rho_{0} \int_{\psi_{1}}^{\psi_{2}}(1-\beta y) d \psi=\rho_{0}-\beta \rho_{0}\left(\psi_{2}^{2}-\psi_{1}^{2}\right) /(2 q) \\
& =\rho_{0}-\frac{\beta \rho_{0}}{2}\left(\psi_{2}+\psi_{1}\right)=\rho_{0}\left(1-\beta \psi_{1}\right)-\frac{\beta \rho_{0}}{2} q,
\end{aligned}
$$


or

$$
\bar{\rho}=\rho_{0}\left(1-\beta y_{s}\right)-\frac{\beta q}{2} \rho_{0},
$$

if again terms of $\mathrm{O}\left(\beta^{2}\right)$ are neglected. The first term on the right-hand side of (40) is the upstream density at $y=y_{s}$. Thus (40) is the general formula containing (39) as a special case.

2.1. Limitations on $F$ and $q$ We call attention to the fact that after a sink has been placed in the stream, the density stratification immediately downstream from the sink will be less than the original one, while the density distribution far downstream will be the original one (even through the velocity far downstream may slow down after the introduction of the sink), so that a density disturbance or density current will develop downstream and may propagate upstream to invalidate the solution. To take care of that, we require $U$ to be greater than the linear wave velocity of the first (fastest) mode in the fluid assumed at rest. In dimensionless terms, this requirement can be shown to be

$$
(1-q) F>1 / \pi
$$

The value of $q$ will be restricted to $1 / 7$. To take care of amplitude effects, we require, liberally,

$$
F>2 / \pi
$$

This condition is not stringent. It does not require $U$ to be more than $1 / 3 \mathrm{~ms}^{-1}$ under realistic conditions (for $\beta$ and $d$ ).

Near the sink there is a region of back flow and density inversion, and if this region is too large, gravitational instability may result. We require that the distance between the sink and the stagnation point downstream from it be only $1 / 20$ of the depth $d$. Then a rough estimate gives the limit

$$
U d / \hat{q}>(40-\pi) / 2 \pi \text { or } q<2 \pi /(40+\pi) .
$$

For simplicity we shall require

$$
q<1 / 7
$$

This is not likely to be exceeded in practice.

2.2. Some numerical results and flow patterns Given $\beta, q$ and $y_{s}$, the average density of the fluid can be computed easily from the formula immediately following (40), and therefore needs no tabulation or graphical representation.

In the problem of pollutant removal, $y_{1}$ and $y_{2}$ may be specified and, for a given $U, y_{s}$ and $q$ are to be determined, as stated in the foregoing. This involves an inverse 
calculation that may require iterations. We choose to exhibit $y_{1}$ and $y_{2}$ for given $\beta, q$, $y_{s}$ and $F$ in Tables 1 to 7. For $y_{s}=0, y_{2}$ can be determined from (9) upon putting its right-hand side equal to $q$ and solving for $y$. The results are given in Table 1 and are independent of $F$. Tables 2, 3 and 4 are for $y_{s}=0.25$. For $\beta=0.01$ and $q=1 / 8$, Table 2 shows that $y_{1}$ and $y_{2}$ are only weakly dependent on $F$ for $F \geq 2 / \pi$. Therefore, in Tables 3 and 4 we have taken $F=3 / \pi$ only. Table 3 shows how $y_{1}$ and $y_{2}$ vary with $q$ for $\beta=0.001$, and Table 4 shows how they vary with $\beta$ for $q=1 / 9$. Correspondingly, for $y_{s}=0.5$, dependence of $y_{1}$ and $y_{2}$ on $F, q$ and $\beta$ is shown in Tables 5,6 and 7. All the tables show that $y_{1}$ and $y_{2}$ are largely independent of $F$ and $\beta$ and dependent only on $q$, for the range of $F$ considered at least.

TABLE 1. $y_{2}$ values with the sink located at $y_{s}=0$.

\begin{tabular}{|c|c|c|c|}
\hline$q$ & $\beta=0.01$ & $\beta=0.05$ & $\beta=0.1$ \\
\hline \hline $1 / 7$ & 0.1430 & 0.1435 & 0.1442 \\
\hline $1 / 8$ & 0.1251 & 0.1255 & 0.1261 \\
\hline $1 / 9$ & 0.1112 & 0.1115 & 0.1120 \\
\hline $1 / 11$ & 0.0910 & 0.0912 & 0.0915 \\
\hline
\end{tabular}

TABLE 2. $y_{1}$ and $y_{2}$ values for $\beta=0.01$ and $q=1 / 8$ with the sink located at $y_{s}=0.25$.

\begin{tabular}{|c|c|c|}
\hline$F$ & $y_{1}$ & $y_{2}$ \\
\hline \hline $2 / \pi$ & 0.2095 & 0.3347 \\
\hline $3 / \pi$ & 0.2055 & 0.3307 \\
\hline $4 / \pi$ & 0.2043 & 0.3295 \\
\hline
\end{tabular}

TABLE 3. $y_{1}$ and $y_{2}$ values for $\beta=0.01$ and $F=3 / \pi$ with the sink located at $y_{s}=0.25$.

\begin{tabular}{|c|c|c|}
\hline$q$ & $y_{1}$ & $y_{2}$ \\
\hline \hline $1 / 11$ & 0.2176 & 0.3087 \\
\hline $1 / 9$ & 0.2104 & 0.3217 \\
\hline $1 / 7$ & 0.1991 & 0.3422 \\
\hline
\end{tabular}

TABLE 4. $y_{1}$ and $y_{2}$ values for $q=1 / 9$ and $F=3 / \pi$ with the sink located at $y_{s}=0.25$.

\begin{tabular}{|c|c|c|}
\hline$\beta$ & $y_{1}$ & $y_{2}$ \\
\hline \hline 0.01 & 0.2104 & 0.3217 \\
\hline 0.05 & 0.2102 & 0.3222 \\
\hline 0.1 & 0.2099 & 0.3227 \\
\hline
\end{tabular}


TABLE 5. $y_{1}$ and $y_{2}$ values for $\beta=0.01$ and $q=1 / 8$ with the sink located at $y_{s}=0.5$.

\begin{tabular}{|c|c|c|}
\hline$F$ & $y_{1}$ & $y_{2}$ \\
\hline \hline $2 / \pi$ & 0.4373 & 0.5627 \\
\hline $3 / \pi$ & 0.4373 & 0.5627 \\
\hline $4 / \pi$ & 0.4373 & 0.5627 \\
\hline
\end{tabular}

TABLE 6. $y_{1}$ and $y_{2}$ values for $\beta=0.01$ and $F=3 / \pi$ with the sink located at $y_{s}=0.5$.

\begin{tabular}{|c|c|c|}
\hline$q$ & $y_{1}$ & $y_{2}$ \\
\hline \hline $1 / 11$ & 0.4544 & 0.5456 \\
\hline $1 / 9$ & 0.4443 & 0.5557 \\
\hline $1 / 7$ & 0.4284 & 0.5716 \\
\hline
\end{tabular}

TABLE 7. $y_{1}$ and $y_{2}$ values for $q=1 / 9$ and $F=3 / \pi$ with the sink located at $y_{s}=0.5$.

\begin{tabular}{|c|c|c|}
\hline$\beta$ & $y_{1}$ & $y_{2}$ \\
\hline \hline 0.01 & 0.4443 & 0.5557 \\
\hline 0.05 & 0.4437 & 0.5562 \\
\hline 0.1 & 0.4429 & 0.5568 \\
\hline
\end{tabular}

Under the limitations (41) and (42), the flow patterns are only weakly dependent on $F$ and $\beta$. We have therefore chosen $F=3 / \pi$ and $\beta=0.01$ in Figures 1,2 and 3 , for three locations of the sink. In these figures $q=1 / 8$. When other values of $q$ satisfying (42) are used, the fluid flowing into the sink becomes thicker or thinner as $q$ increases or decreases, but the general flow pattern is otherwise little changed. The lack of dramatic changes in the flow pattern as $F$ and $q$ are varied within the limitations of (41) and (42) indicates that in a flowing stratified fluid (or for a moving sink) control of selective withdrawal is certain and entirely at our disposal.

\section{Analysis for other upstream velocities}

For comparison with the results obtained for the upstream velocity distribution given by (8), let us now keep the total discharge $U d+\hat{q}$ and the limitations on $F$ and $q$, but assume

$$
u=C\left(\rho_{0} / \rho\right)^{1 / 2}, \quad \text { or } \quad \psi^{\prime}=C y .
$$




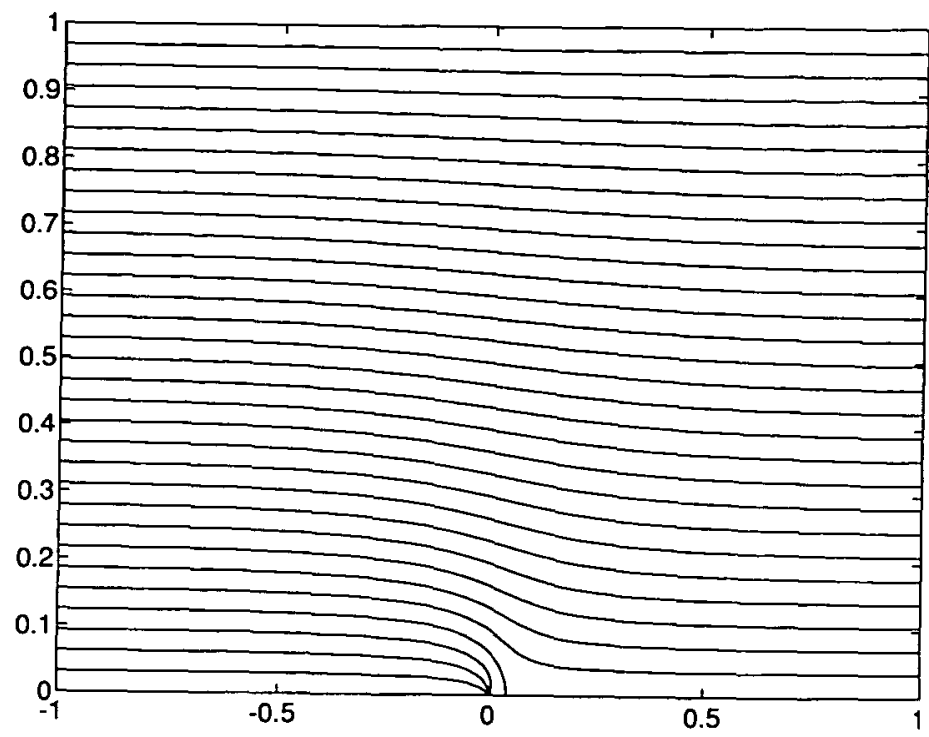

FIGURE 1. The flow pattern when the sink is located at $y_{s}=0(\beta=0.01, q=1 / 8$ and $F=3 / \pi)$.

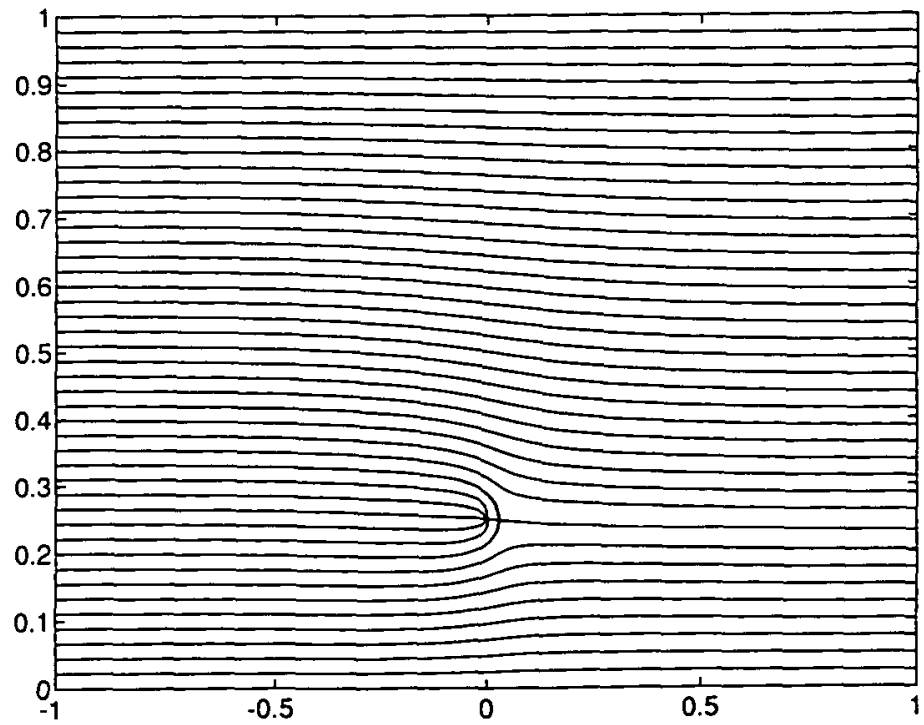

FIGURE 2. The flow pattern when the sink is located at $y_{s}=0.25(\beta=0.01, q=1 / 8$ and $F=3 / \pi)$.

far upstream. It can be verified that the total discharge is maintained if

$$
C=\frac{\beta\left[1-(1-\beta)^{1 / 2}\right]^{-1}}{2}=1-\beta / 4+\mathrm{O}\left(\beta^{2}\right)
$$




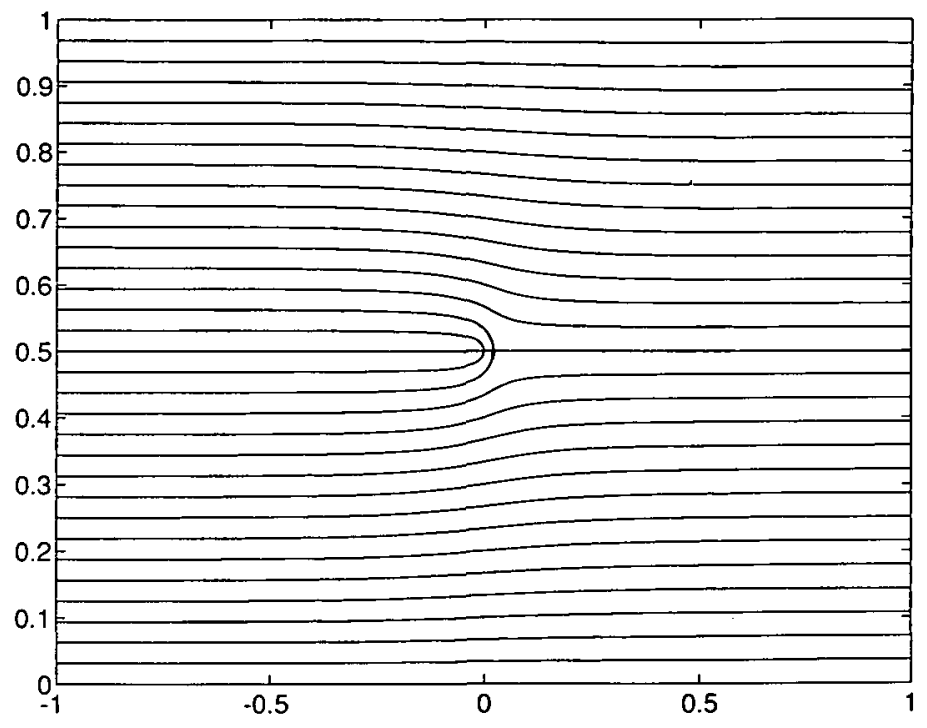

FIGURE 3. The flow pattern when the sink is located at $y_{s}=0.5(\beta=0.01, q=1 / 8$ and $F=3 / \pi)$.

Then (17) becomes, upon determination of $h\left(\psi^{\prime}\right)$,

$$
\nabla^{2} \psi^{\prime}-F^{-2} C^{-1} y=-F^{-2} C^{-2} \psi^{\prime}
$$

Writing $\psi^{\prime}=C y+\phi$, we have

$$
\nabla^{2} \phi+F^{-2} C^{-2} \phi=0
$$

With the new densimetric Froude number $\tilde{F}$ defined by

$$
\tilde{F}=C F,
$$

(45) can be written as

$$
\nabla^{2} \phi+\tilde{F}^{-2} \phi=0
$$

The rest of the analysis is the same as in Section 2. Since (13) and (43) differ by terms of $O(\beta)$ and so do (24) and (47), it is clear that (39) and (40) are unaffected, and the determination of $y_{2}$ and $y_{1}$ will give results that differ from those determined in Section 2 by quantities of $O(\beta)$ only.

If we use the upstream velocity given by Yih's theorem [11] based on shallowwater theory, the same situation is obtained. In that paper, a transformation is used to connect the actual velocity $(u, v)$ with the transformed velocity $(U, V)$ for a uniform fluid with the same flow pattern. That transformation is

$$
(u, v)=\lambda(\rho)(U, V), \quad \lambda^{2}=B(\rho),
$$


where $B(\rho)$ is a function of $\rho$ alone, determined by the density distribution in the reservoir where the flow originates. In the present case $B(\rho)$ is determined from a linear density stratification in the reservoir, with the gradient $\beta$. [The density distribution in the channel after the fluid issues from the reservoir is then given by (2), with a linear quantity $\mathrm{O}\left(\beta^{2}\right) y$ neglected.] Thus, we have

$$
B(\rho)=1-\beta / 2+\beta y-\beta y^{2} / 2+\mathrm{O}\left(\beta^{2}\right),
$$

so that the $\lambda$ defined in that paper is

$$
\lambda=1-\beta / 4+\beta y / 2-\beta y^{2} / 4+\mathrm{O}\left(\beta^{2}\right) .
$$

Then if we take the $U$ in that paper to be $1+\beta / 12$ far upstream, in order to make the total dimensionless discharge equal to 1 , we have the upstream velocity

$$
u=(1+\beta / 12) \lambda=1-\beta / 6+\beta y / 2-\beta y^{2} / 4,
$$

upon neglect of $\mathrm{O}\left(\beta^{2}\right)$. The rest of the analysis is the same as that in Section 2, and $\phi$ is again governed by (47) if terms of $\mathrm{O}(\beta q)$ are neglected and

$$
\tilde{F}=(1-\beta / 6) F \text {. }
$$

Thus, accepting errors of $\mathrm{O}\left(\beta^{2}\right)$ for $\bar{\rho}$ and of $\mathrm{O}(\beta)$ for $y_{2}$ and $y_{1}$ and the flow pattern, the results obtained in Section 2, or by using the simpler upstream condition (43), are the required results for the upstream velocity given by (48).

The theorem of Yih [11] was not an extension of any special results for two homogeneous layers, but was a completely new result for any density stratification. Yih did not claim that all channel flows issuing from a large reservoir have the velocity distributions given by his theorem, because that simply is not true, any later claims by others to the contrary notwithstanding. A high barrier downstream, such as a vertical boundary above a sink located at the bottom, would block part of the fluid at low densimetric Froude numbers, and give rise to a stagnant layer extending all the way back to the reservoir, thereby drastically invalidating the upstream velocity distribution in the channel given by that theorem. Selective withdrawal from an otherwise quiescent stratified fluid, explicitly or implicitly studied by all the authors cited in the introduction, is, after all, an exploitation of the phenomenon of blocking. The upstream influence of downstream geometry cannot be ignored. In the present problem, the underlying assumptions of the shallow-water theory are violated near the sink. But this violation probably does not have enough upstream influence to invalidate (48). In any event, our purpose was to show that any slight variation from uniformity in the upstream velocity has a negligible effect on the results. 


\section{The use of $\psi^{\prime}$}

From the foregoing it is clear that, after the associated stream function $\psi^{\prime}$ has been introduced to take care of convective acceleration once and for all, the flow pattern, the result for $\bar{\rho}$ of the fluid withdrawn and the other results of interest depend very little on any slight variation in the upstream velocity.

But this is not to say that the inertial effect of density variation is negligible. If one adopts the Boussinesq approach and assumes the density to be constant $\left(=\rho_{0}\right)$ where it is multiplied to the acceleration, but not where it is multiplied by $g$, one can obtain a dimensionless equation

$$
\nabla^{2} \psi+F_{0}^{-2} \frac{d\left(\rho / \rho_{0}\right)}{d \psi} y=h(\psi)
$$

which has exactly the form of (17), except that $\psi$ has replaced $\psi^{\prime}$. Working with this equation and assuming a uniform velocity far upstream, one can determine $h(\psi)$ and obtain substantially the same results as presented in Section 2 or 3 . One is then tempted to say that the inertial effect of density variation is unimportant, and the introduction of $\psi^{\prime}$ in the analysis is unnecessary and therefore merely pedantic. But in neglecting the inertial effect of density variation in the present problem, the Boussinesq equation is seriously in error near the sink, where the velocity and acceleration are very large and the inertial effect of density variation very great.

\section{Conclusions}

The results and their discussion presented above need not be repeated here. The main conclusion of this paper is that selective withdrawal from a stratified fluid, be its purpose control of the average temperature or salinity of the fluid withdrawn or removal of pollutants, can certainly be achieved in a flowing stream or by a moving sink, under the stated limitations on the (densimetric) Froude number and the discharge into the sink. The thickness and location of the withdrawn layer are largely independent of the Froude number and the density gradient, and depend mainly on the strength and location of the sink. The difficulties encountered (see Yih, [9]) in attempts at selective withdrawal by a stationary sink at high (that is, $>1 / \pi$ ) Froude numbers from an otherwise quiescent fluid are thus avoided.

\section{References}

[1] A. Craya, "Recherches théoriques sur l'écoulement de couches superposées de fluides de densités différentes", La Houille Blanche 4 (1949) 44-55. 
[2] W. R. Debler, "Stratified flow into a line sink", J. Eng. Mech. Div., Proc. Am. Soc. Civ. Eng. 85 (1959) 51-65.

[3] P. Gariél, "Recherches expérimentales sur l'écoulement de couches superposées de fluides de densités différentes", La Houille Blanche 4 (1949) 56-64.

[4] G. C. Hocking, "Cusp-like free surface flows due to a submerged source or sink in the presence of a flat or slopping bottom", J. Austral. Math. Soc. Ser, B 26 (1985) 470-486.

[5] T. W. Kao, "Free-streamline theory for inviscid stratified flow into a line sink", Phys. Fluids 13 (1970) 558-564.

[6] A. R. Richardson, "Stationary waves in water", Philos. Mag. 40 (1920) 79-110.

[7] E. O. Tuck and J.-M. Vanden-Broeck, "A cusp-like free-surface flow due to a submerged source or sink", J. Austral. Math. Soc. Ser. B 25 (1984) 443-450.

[8] J.-M. Vanden-Broeck and J. B. Keller, "Free surface flow due to a sink", J. Fluid Mech. 175 (1987) 109-117.

[9] C.-S. Yih, "On the flow of a stratified fluid", Proc. 3rd U.S. Nat. Congr. Mech. (1958) 857-861.

[10] C.-S. Yih, "Exact solutions for steady two-dimensional flow of a stratified fluid", J. Fluid Mech. 9 (1960) 161-174.

[11] C.-S. Yih, "A Class of solutions for steady stratified flows", J. Fluid Mech. 36 (1969) 75-86. 